\title{
DISSECTING THE GRB ENVIRONMENT WITH OPTICAL AND X-RAY OBSERVATIONS
}

\author{
S. Campana ${ }^{1}$
}

\begin{abstract}
GRB's environment can be studied through the imprint (i.e. absorption) it leaves on the afterglow emission. Fast optical observations allowed us to observe line variability and to derive the distance and composition of the absorbing medium. However, given the huge GRB ionizing flux, this medium starts showing up in the optical spectra only at relatively large distances ( $\gtrsim 100 \mathrm{pc})$. A complementary view comes from the X-ray band, where the overall contribution of all metals bends the power law X-ray afterglow spectrum. We will review and compare optical and X-ray studies of the circumbust medium.
\end{abstract}

\section{Introduction}

Long duration Gamma-ray Bursts (GRBs) are associated with the death of massive stars. This evidence comes principally from the association of close-by GRBs $(z \lesssim 0.5)$ to type Ic Supernovae (Woosley \& Bloom 2006). In addition, GRBs occur in the most luminous part of their host galaxies (Svensson et al. 2010). These observational facts clearly point to a close relationship between star formation and GRBs, suggesting that the ambient medium in which GRBs explode is denser than the interstellar medium and typical of star forming regions. In this contribution we will focus on the properties of the ambient medium that can be envisaged through optical and X-ray studies.

\section{Dark GRBs}

Since the discovery of GRB afterglows it has become clear that a number of GRBs lack an optical afterglow (Fynbo et al. 2001). Given the different observing conditions, a more rigorous definition is needed besides the "no optical afterglow" statement. Based on the predictions of the fireball model (Mészáros \& Rees 1997)

1 INAF - Osservatorio astronomico di Brera, via Bianchi 46, 23807 Merate (LC), Italy; e-mail: sergio.campana@brera.inaf.it 
one can require that the optical to X-ray spectral index $\beta_{O X}$ (i.e. the slope between the fluxes in the $R$-band and at $1 \mathrm{keV}$ ) should be lower than 0.5 (Jakobsson et al. 2004). This will characterize optically sub-luminous bursts, i.e. fainter than expected from the fireball model. With the advent of the Swift satellite, X-ray spectral slopes were available and a somewhat different definition was put forward by van der Horst et al. (2009) for which $\beta_{O X}$ is shallower than $\beta_{X}-0.5$.

Earlier studies suggested that $20-50 \%$ range of GRBs are dark, and dust absorption, high redshift or intrinsically dim afterglows are possible causes. With the advent of complete samples of GRBs this matter can be settled on more solid grounds. The flux limited Swift-BAT6 sample (Salvaterra et al. 2012) and the GROND sample (Greiner et al. 2011) both provide a fraction of $25-40 \%$ of dark GRBs (Melandri et al. 2012) and likely indicate dust absorption (possibly helped in some cases by a relatively large redshift) as their likely cause. At variance with earlier studies also the distribution of optical absorptions, $A_{V}$, in complete samples of GRBs is not negligible, showing a continuum of values from zero to a few (Covino et al. 2013; Greiner et al. 2011, see Fig. 1a).

A clearer picture arises from X-ray studies where a connection between the intrinsic (in situ) absorbing column density and the GRB darkness was found. Comparing the intrinsic column density distribution of dark and non-dark GRBs of the Swift-BAT6 sample, a Kolmogorov-Smirnov test returns a probability of $2 \times$ $10^{-6}$ (corresponding to $4.8 \sigma$ ) for Jakobssons darkness definition and $1 \times 10^{-5}(4.4 \sigma)$ for van der Horsts definition. These results indicate that the intrinsic absorption as evaluated in the X-ray band is highly correlated with the darkness of a GRB (Campana et al. 2012, see Fig. 1b).

\section{Optical spectroscopy}

Optical absorption $\left(A_{V}\right)$ and X-ray column density $\left(N_{H}\right)$ provide a cumulative (integral) measure of the matter along the line of sight. Deeper insight comes from (high resolution) spectroscopic studies. These studies reveal that the absorption lines relative to the GRB site is made up by several components and trace the distribution of matter along the line of sight within the host galaxy (in addition to lines coming from intervening systems). The strongest lines are CII, CIV, OI, SiII, SiIV, MgI and FeII and are characterized by blue as well as red-shifts, spanning up to $\pm 150 \mathrm{~km} \mathrm{~s}^{-1}$. These lines are interesting and provide a clue on the number of absorbing systems as well as on the metallicity of the host galaxy, but we can have only an indirect idea of their distances from the GRB site. Indirect modeling based on ionization potential and burst flux can lead to estimate a minimal distance from the GRB of $>50-100 \mathrm{pc}$, for e.g. the MgI line in GRB 051111 (Prochaska et al. 2005).

The key element to assess the absorption systems' distances is line variability. The first line variation was observed in GRB 020813, comparing a Keck/LRIS spectrum obtained $\sim 5 \mathrm{hr}$ after the GRB event with a VLT/UVES spectrum acquired $\sim 16 \mathrm{hr}$ after the first one: a FeII fine-structure transition $(J=7 / 2)$ was clearly detected to vary (Dessauges-Zavadsky et al. 2006). The line variability 

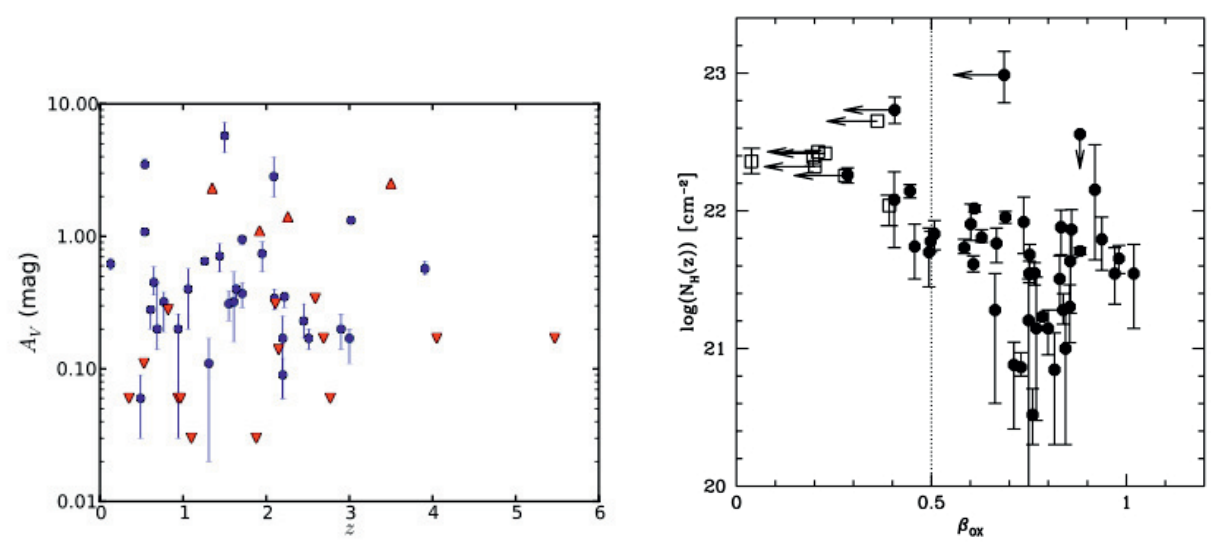

Fig. 1. Left panel: redshift dependence of $A_{V}$ in the complete Swift-BAT6 sample (from Covino et al. 2013). The $A_{V}$ have been estimated through Spectral Energy Distribution fitting. Right panel: column density $\left(N_{H}\right)$ as a function of the spectral index $\beta_{O X}$ for the Swift-BAT6 GRBs computed in Melandri et al. (2012). The dashed line for $\beta_{O X}=0.5$ divides dark and non-dark GRBs according to Jakobsson et al. (2004). The open squares (filled circles) indicate dark (non-dark) GRBs according to van der Horst et al. (2009, from Campana et al. 2012).

technique was first exploited with GRB 060418. For this burst the VLT Rapid Response Mode (RRM) was activated producing spectra at 13, 15, 20, 30 and 90 min after the burst onset. Fine structure variability in six FeII lines were clearly detected (together with Ni II; Vreeswijk et al. 2007, Fig. 2a). Modeling of these line variations led us to exclude collisional excitation and IR excitation as viable mechanisms. On the contrary, UV pumping (powered by the GRB UV flux) was found to be the dominant mechanism and led to estimate the absorbing region distance to be $0.5 \pm 0.1 \mathrm{kpc}$. The best example is provided by the naked-eye burst GRB 080319B observed with VLT/UVES in RRM (see Fig. 2b). Six different absorption systems were identified within the host galaxy, spanning a distance range of $0.6-1.7 \mathrm{kpc}$ (D'Elia et al. 2009). In addition to fine structure variability, in only one case a variability (decrease) in the Lyman $\alpha$ line intensity was detected. The optical spectra of GRB 090426 taken at 1 and $12 \mathrm{hr}$ after the burst, show a $3.8 \sigma$ decrease in the line Equivalent Width. This is the first compelling evidence for photoionization of the material from the star-forming region of the progenitor itself. Based on a tailored photoionization code the distance of the absorber has been estimated in $\sim 80$ pc (Thöne et al. 2011, Fig. 3a).

A summary of all the absorption systems distances in GRB afterglow spectra is shown in Figure 3b. These are all derived from fine structure line variations apart from GRB 090426. Distances range from 80 pc to a few kpc. This scale is comparable to the expected size of the shock among the progenitor's wind and the Interstellar Medium (ISM). All the material within $\sim 100 \mathrm{pc}$ is heavily affected by the GRB photo-ionizing flux. 

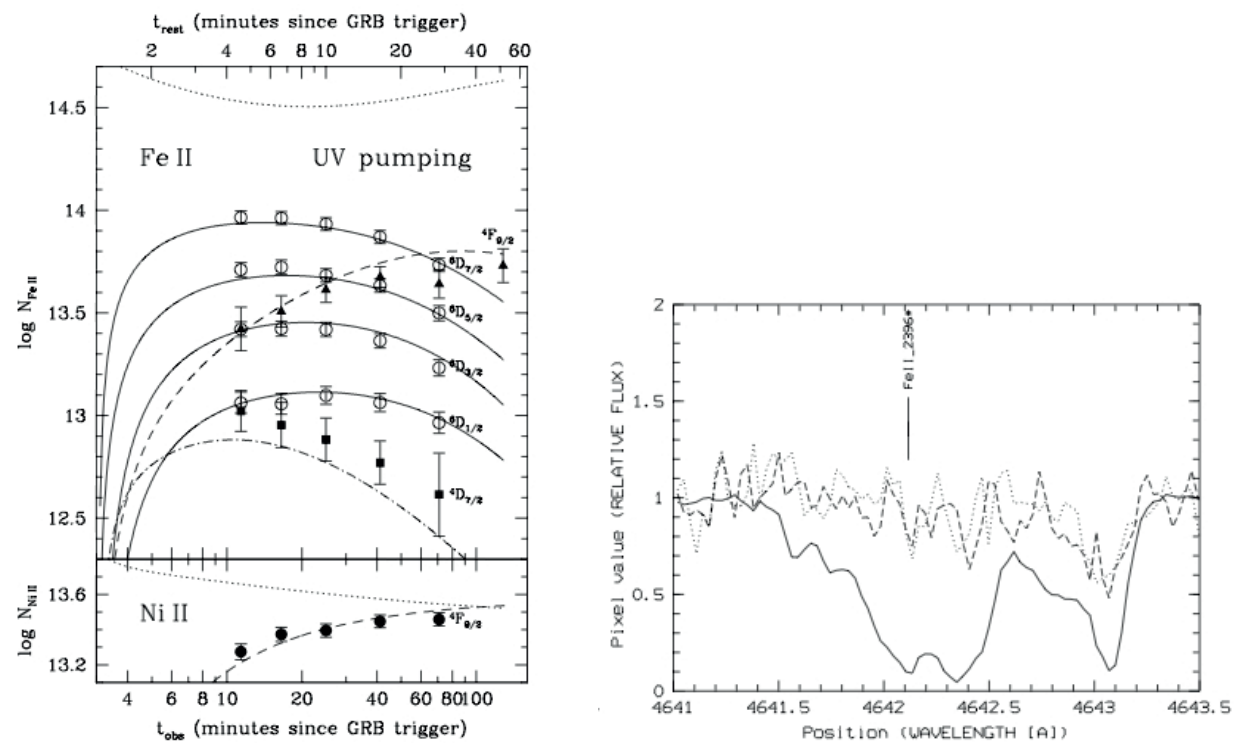

Fig. 2. Left panel: Fe II and Ni II column density variations observed in GRB 060418 with several VLT/UVES observations. Line variations are very well described by the UV pumping model (from Vreeswijk et al. 2007). Right panel: UVES spectra of GRB 080319B around the fine-structure FeII $\lambda 2396^{*}$ line. The solid line refers to the 1st epoch spectrum ( $8.5 \mathrm{~min}$ after the trigger), the dashed line refers to the 2 nd spectrum $(1.9 \mathrm{hr})$, and the dotted line to the 3rd spectrum (2.9 hr; from D'Elia et al. 2009).

\section{X-ray observations}

At optical wavelengths the GRB flux is too strong and no line variations are observed to arise within $\sim 100 \mathrm{pc}$. To probe the medium closer to the progenitor, one can look at higher ionization lines, like $\mathrm{S}^{+4}, \mathrm{~N}^{+5}$, and $\mathrm{O}^{+6}$. These lines are observed (sometimes) in GRB optical spectra but, up to now, no variations were revealed. As a matter of fact at optical wavelengths we are unable to dissect the closest medium to the GRB. This is due to the high GRB photoionization flux. Ideally we can try to study the medium at even shorter wavelengths, hoping that the GRB ionizing flux effects are reduced. Given the spectral resolution and effective area of current X-ray facilities it is difficult to reveal line or absorption edge variations in the X-ray spectra of GRBs (unless possibly with the Reflection Grating Spectrometers on board XMM-Newton). Along these lines one can try to study (X-ray) column density variations, i.e. the integrated absorption caused by metals surrounding the GRB site.

A study of the intrinsic absorbing column density in the complete sample of Swift-BAT6 bursts shows that these events are heavily absorbed with a mean absorption of $\log \left(N_{H} / \mathrm{cm}^{-2}\right)=21.7 \pm 0.5$ (Campana et al. 2012). In addition, the distribution is consistent with the expected distribution obtained by putting 

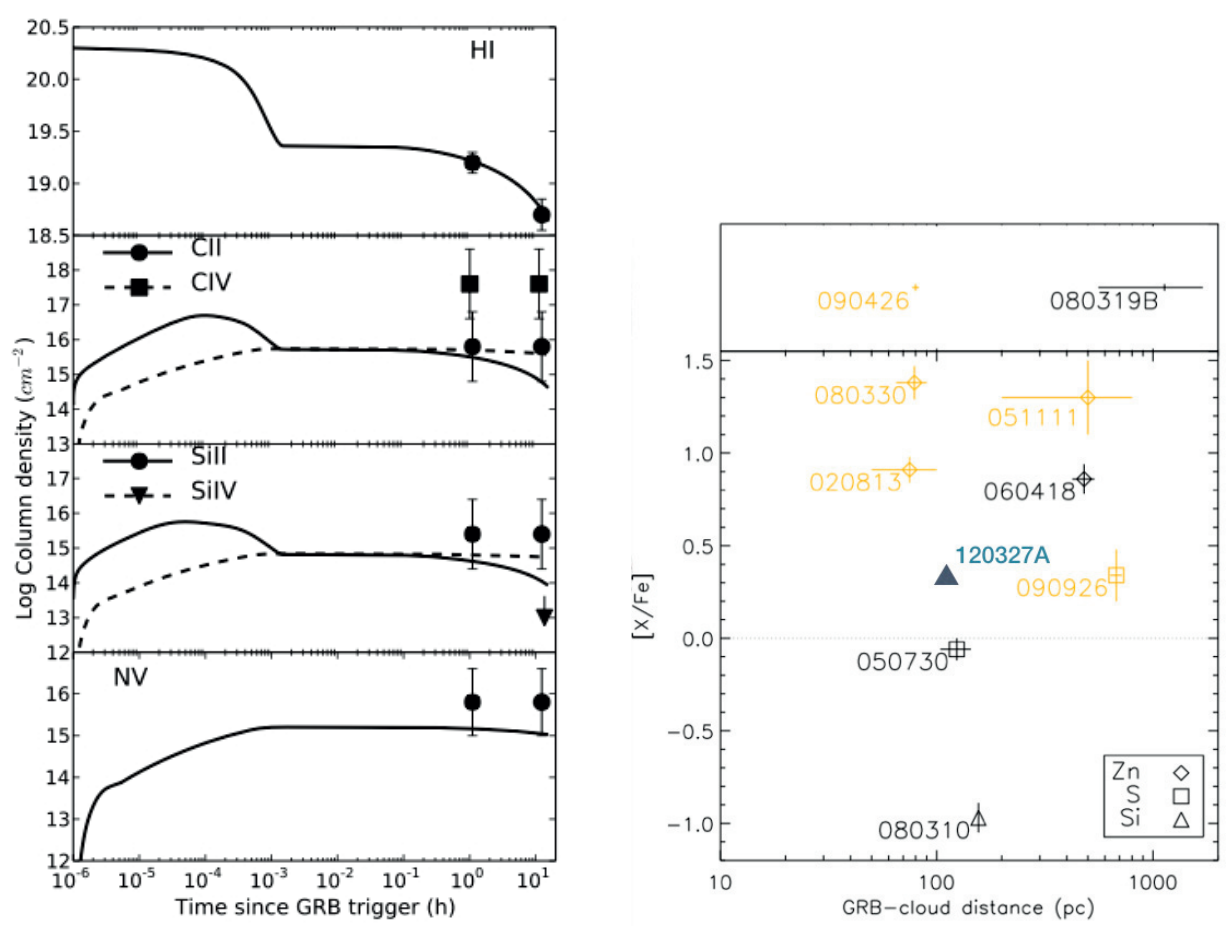

Fig. 3. Left panel: temporal evolution of the absorbing columns of $\mathrm{H}, \mathrm{C}, \mathrm{Si}$ and $\mathrm{N}$ ions. The best-fitting evolution of the absorbing columns as derived from our photoionization model is overlaid (from Thöne et al. 2011). Right panel: summary of absorbing system distances in GRB afterglow optical spectra (from Vreeswijk et al. 2012).

GRBs randomly within Giant Molecular Clouds similar to the ones observed in our Galaxy (Campana et al. 2010, 2006). This testifies once more that GRBs occur in dense regions. A comparison between optical and X-ray column densities shows interesting results. After correcting for metallicities, X-ray absorbing columns are always larger than optical columns $\left(N_{H I}\right)$ by a factor of $\sim 10$ (but in some cases much more, see Fig. 4a). This is can be interpreted as a manifestation of GRB photoionization (Campana et al. 2010; Watson et al. 2007).

Column density variations are needed to assess the absorber distance. These are difficult to detect due to the spectral changes associated to the afterglow evolution. In the high-redshift GRB 050904 a column density variation has been detected by several groups (Watson et al. 2006; Boër et al. 2006; Gendre et al. 2006; Campana et al. 2007). This has been modeled with a photoionization code leading to the characterization of the absorbing medium (Fig. 4b). The absorbing region is estimated to lie very close to the GRB site, some 5-10 pc away (Campana et al. 2007). In addition, the optical transient was observed at $t_{\text {obs }} \sim 200 \mathrm{~s}$ (i.e. $27 \mathrm{~s}$ rest frame) in white light, indicating very little absorption. The little extinction implied by the early optical observation can be explained by a dust component 

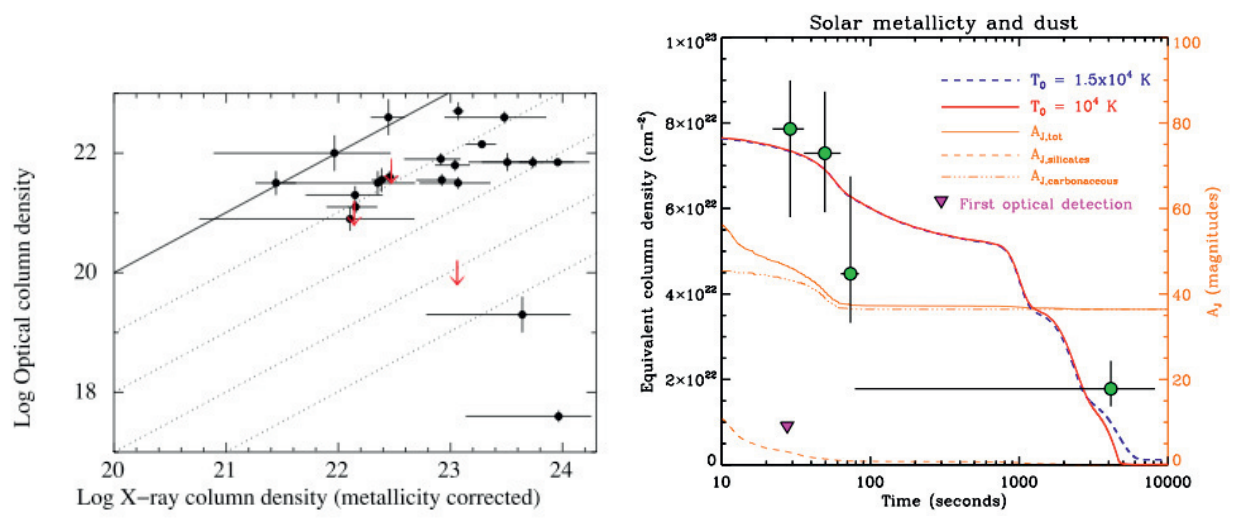

Fig. 4. Left panel: X-ray (metallicity corrected) versus optical column densities for GRBs. The continuous line refers to equal optical to X-ray values, the dotted lines to $n$-th orders of magnitude difference (from Campana et al. 2010). Right panel: evolution of the column density measured in the X-ray afterglow of GRB 050904 (circles with error bars at $1 \sigma)$. Time is in the rest frame $(z=6.4)$. The thick solid and the thick dashed lines show the best-fit models for different initial temperatures. The photoionization code has in input the observed light curve of GRB 050904 (Cusumano et al. 2006). The drop in absorption at $t \sim 1000 \mathrm{~s}$ (rest frame) corresponds to a group of bright X-ray flares. The thin solid line (and right y-axis) shows the amount of absorption that would be observed in the $J$ band (rest frame $7 \mathrm{eV}$ ) if the X-ray absorbing medium were polluted with Galactic-like dust. Thin dashed and dot-dashed lines show the absorption due to silicates only and to carbonaceous grains only, respectively (from Campana et al. 2007).

rich in silicates and depleted in carbonaceous grains. This could be the result of an ISM enriched by pair-instability supernovae. The involved mass is very large but it is in line with the medium density of $\sim 700 \mathrm{~cm}^{-3}$, estimated through radio studies (Frail et al. 2006).

\section{Conclusions}

The ambient medium surrounding GRBs is complex but it holds the potential to unveil the progenitor's latest stages and nature. There is strong evidence to connect (long) GRBs to star formation and their formation site to dense ambient media. Optical high-resolution spectroscopic studies can dissect the GRB environment in exquisite detail, sampling however regions larger than $\sim 100 \mathrm{pc}$. To probe the ambient medium closer to the progenitor, we need to trace higher energy transitions, thus reducing the influence of the huge GRB photoionization flux. This cannot be done with current facilities, lacking a fast-slewing, high spectral resolution X-ray telescope. An integral description of the X-ray absorption $\left(N_{H}\right)$ can be in any case gathered thanks to the Swift/XRT instrument and in a few cases $N_{H}$ variations enable us to probe the absorbing medium in the proximity of the GRB progenitor. 
This work has been supported by ASI grant I/004/11/0. The number of people I have to thank is very large so I will put just initials: MGB, SC, GC, PDA, VDE, MDV, GG1, GG2, DL, DM, AM1, AM2, LN, NP, PR, RS, BS, GT, CT, SDV.

\section{References}

Boër, M., Atteia, J.L., Damerdji, Y., et al., 2006, ApJ, 638, L71

Campana, S., Mangano, V., Blustin, A.J., et al., 2006, A\&A, 454, 113

Campana, S., Lazzati, D., Ripamonti, E., et al., 2007, ApJ, 654, L17

Campana, S., Thöne, C.C., de Ugarte Postigo, A., et al., 2010, MNRAS, 402, 2429

Campana, S., Salvaterra, R., Melandri, A., et al., 2012, MNRAS, 421, 1697

Covino, S., Melandri, A., Salvaterra, R., et al., 2013, MNRAS, submitted

Cusumano, G., Mangano, V., Chincarini, G., et al., 2006, Nature, 440, 164

D'Elia, V., Fiore, F., Perna, R., et al., 2009, ApJ, 694, 332

Dessauges-Zavadsky, M., Chen, H.-W., Prochaska, J.X., et al., 2006, ApJ, 648, L89

Frail, D., Cameron, P.B., Kasliwal, M., et al., 2006, ApJ, 646, L99

Fynbo, J.P.U., Gorosabel, J., Dall, T.H., et al., 2001, A\&A, 373, 796

Gendre, B., Galli, A., Corsi, A., et al., 2007, A\&A, 462, 565

Greiner, J., Krühler, T., Klose, S., et al., 2011, A\&A, 526, A30

Jakobsson, P., Hjorth, J., Fynbo, J.P.U., et al., 2004, A\&A, 427, 785

Melandri, A., Sbarufatti, B., D'Avanzo, P., et al., 2012, MNRAS, 421, 1265

Mészáros, P., \& Rees, M.J., 1997, MNRAS, 288, L51

Prochaska, J.X., Chen, H.-W., \& Bloom, J.S., 2005, ApJ, 648, 95

Salvaterra, R., Campana, S., Vergani, S.D., et al., 2012, ApJ, 749, 68

Svensson, K.M., Levan, A.J., Tanvir, N.R., et al., 2010, MNRAS, 405, 57

Thöne, C.C., Campana, S., Lazzati, D., et al., 2011, MNRAS, 414, 479

van der Horst, A.J., Kouveliotou, C., Gehrels, N., et al., 2009, ApJ, 699, 1087

Vreeswijk, P.M., Ledoux, C., Smette, A., et al., 2007, A\&A, 468, 83

Vreeswijk, P.M. Ledoux, C., De Cia, A., \& Smette, A., 2012, MSAIS, 21, 14

Watson, D., Reeves, J.N., Hjorth, J., et al., 2006, ApJ, 637, L69

Watson, D., Hjorth, J., Fynbo, J.P.U., et al., 2007, ApJ, 660, L101

Woosley, S.E., \& Bloom, J.S., 2006, ARA\&A, 44, 507 
